\title{
Numerical simulation of tensile tests of prestrained sheets
}

\author{
L.F. Menezes *, J.V. Fernandes, D.M. Rodrigues \\ Departamento de Engenharia Mecânica, CEMUC, Universidade de Coimbra Pólo II, Pinhal de Marrocos, 3030 Coimbra, Portugal \\ Received 20 July 1998; received in revised form 26 October 1998
}

\begin{abstract}
The effect of cross section variation on formability of prestrained samples has been investigated using finite element simulations of a standard sheet tensile test. The mechanical model takes into account large elastoplastic strains and rotations that occur during deformation. Hill's orthotropic yield criterion with isotropic hardening describes the anisotropic plastic properties of the sheet. The isotropic hardening is modelled by a modified Swift law that describes the response of prestrained materials in reloading. Two different situations were simulated: reloading in tension of samples with constant cross sectional area and reloading in tension of samples with two zones of slightly different cross sectional areas. The results show that the strain distribution along the tensile axis of a prestrained sample depends on the level of the prestrain and also on the presence and size of geometrical fluctuations in the cross section, which always occur in experimental samples. This dependence is higher for materials with lower work-hardening rates. (C) 1999 Elsevier Science S.A. All rights reserved.
\end{abstract}

Keywords: Mechanical modelling; Numerical simulation; Plasticity

\section{Introduction}

The plastic flow behaviour of metals during sequential strain paths has been exhaustively analyzed in the past few years (see for example [1-18] and references therein). The aspects of mechanical behaviour associated with the strain path changes are now well known. In general, the mechanical behaviour of metals after path changes follows the following pattern: the higher the initial flow stress in reloading the lower the workhardening rate and the total uniform plastic deformation, during the complex path. In a previous work [19], the authors have formulated an empirical equation that accurately describes the experimental stress-strain curves of prestrained samples. This is a Swift type equation that, taking into account the monotonic behaviour in tension and the values of the normalized reloading yield stress, predicts the work-hardening behaviour after prestrain.

The non-uniform deformation sometimes observed at the beginning of the reloading in tension, before the

\footnotetext{
* Corresponding author. Tel.: + 351 39790700; fax: + 351 39790701.

E-mail address: luis.menezes@mail.dem.uc.pt (L.F. Menezes)
}

maximum load is attained, is not connected with local instability at the grain level [17]. The experimental analyses indicates that this effect consists of a delay in starting deformation in some regions, whose deformations evolve differently along the sample and must be related with the mechanical behaviour and the presence of geometrical defects. In order to get a better understanding of this effect, further analyses is needed. However, the experimental measurement of the evolution of the strain distribution along the samples, during tensile tests, needs specific analysis that is not simple to perform with common test equipment. In this context, the numerical simulation is a powerful alternative tool if correct mechanical models are used.

In the present work, the modified Swift equation is implemented in a three-dimensional finite element based code developed for the numerical simulation of problems with large plastic deformation [20]. The mechanical model involved takes into account the large elastoplastic strains and rotations that occurs in these processes. Hill's orthotropic yield criteria with isotropic hardening describes the anisotropic plastic properties of the material. The hardening is modelled by the new equation. The code makes use of a fully implicit algorithm of Newton-Raphson type to solve the incre- 
mental problem. To initialize the iterative loop, a static explicit algorithm associated with an automatic choice of the incremental step calculates a first approach of the solution. Spatial discretization is made with threelinear eight-node isoparametric 'brick' elements. A selective reduced integration technique is used to overcome the problem of locking associated with these elements.

The code was used to simulate the uniaxial tensile test in reloading of sheet samples with different prestrained values. The parameter used in the modified Swift law, that depends on the path change, was previously known. So, the path change itself is not part of the simulation. Several simulations were made to emphasize the influence of geometrical fluctuations of the cross-sectional area on the strain distribution along the length of the tensile samples.

\section{Mechanical modelling}

The evolution of the deformation process is described by using an updated Lagrangian scheme, i.e. the configuration of the deformed body at time $t$ is taken as a reference configuration for the time interval $[t, t+\Delta t], \Delta t$ being the time increment. After this, the configuration and the state variables are updated and the result is taken as the reference configuration during the subsequent time increment.

The mathematical formulation associated with the elastoplastic behaviour of the material and with the mechanical model are described in this section. In the following, a superposed dot denotes the material time differentiation. The summation convention over repeated indices is used throughout the paper.

\subsection{The constitutive law}

For small elastic strains, the isotropic Hooke's law implies that:

$D_{i j}^{e}=\frac{1}{E}\left[(1+v) \dot{\sigma}_{i j}^{J}-v \dot{\sigma}_{m m} \delta_{i j}\right]$,

where $\boldsymbol{D}^{e}$ is the elastic part of the strain rate tensor $\mathbf{D}$, $E$ is the Young modulus, $v$ is the Poisson ratio and is the Jaumann derivative of the Cauchy stress tensor $\boldsymbol{\sigma}$.

The plastic behaviour is described by the general quadratic yield condition:

$f(\bar{\sigma}, Y)=\bar{\sigma}-Y(h)=0$,

and by the associated inviscid flow rule:

$D_{i j}^{p}=\dot{\lambda} \frac{\partial f}{\partial \sigma_{i j}}$,

where $Y$ is the flow stress in simple traction which is a function of the scalar parameter $h$ that represents the isotropic work-hardening. $\dot{\boldsymbol{D}}^{p}$ is the plastic part of the strain rate tensor and $\dot{\lambda}$ is the plastic multiplier. $\bar{\sigma}$ is the equivalent stress that is defined by the quadratic form:

$\bar{\sigma}^{2}=\boldsymbol{\sigma}: \mathbf{M}: \boldsymbol{\sigma}$

where $\boldsymbol{M}$ is a fourth-order symmetric tensor where evolution must be taken into account during the deformation process. To perform uniaxial tensile tests in sheet specimens we can suppose that the material is initially orthotropic and that it remains so during the deformation. However, the assumption of orthotropic symmetry only makes sense if the tensile axis is parallel to one of the orthotropic axes (rolling or transverse direction of the sheet). The orthotropic axes are supposed to coincide initially with the Cartesian axes $x_{i}$ and to be subjected to a time-dependent rotation $\boldsymbol{R}$. Then, the initial anisotropy tensor, say $\hat{\boldsymbol{M}}$, corresponds to Hill's quadratic yield condition and:

$$
\begin{aligned}
\bar{\sigma}^{2}= & \hat{M}_{i j k m} \sigma_{i j} \sigma_{k m} \\
= & F\left(\sigma_{22}-\sigma_{33}\right)^{2}+G\left(\sigma_{33}-\sigma_{11}\right)^{2}+H\left(\sigma_{11}-\sigma_{22}\right)^{2}+2 L \sigma_{23}^{2} \\
& +2 M \sigma_{13}^{2}+2 N \sigma_{12}^{2}
\end{aligned}
$$

where $F, G, H, L, M$ and $N$ are material constants. Then, the evolution of $\boldsymbol{M}$ is simply given by:

$M_{i j k m}=R_{i n} R_{j p} R_{k q} R_{m s} \hat{M}_{n p q s}$,

and the evolution of $\boldsymbol{R}$ is governed by the differential equation:

$\dot{\boldsymbol{R}}=\left(\boldsymbol{W}-\boldsymbol{W}^{p}\right) \boldsymbol{R}$,

where $\boldsymbol{W}$ is the rotation rate tensor and $\boldsymbol{W}^{p}$ is its plastic part (plastic spin) that we can assume to be zero $\left(\boldsymbol{W}^{p} \simeq 0\right)[21]$. The orthotropy frame is then supposed to turn together with the material.

In Eq. (2), the function $Y(h)$ commonly used is a Swift equation of the type:

$$
Y(\varepsilon)=C\left(\varepsilon_{0}+\varepsilon\right)^{n},
$$

where $C, n$ and $\varepsilon_{0}$ are constants for a particular material, determined in uniaxial tension tests. Based on this equation, a modified equation for prestrained materials has been formulated as follows [19]:

$Y\left(\varepsilon^{*}\right)=C\left[g\left(\varepsilon_{0}+\bar{\varepsilon}_{p}\right)+h \varepsilon^{*}\right]^{n}$.

In this equation $\bar{\varepsilon}_{p}$ is the von Mises equivalent prestrain value and $\varepsilon^{*}$ is the plastic strain in reloading. The parameter $g$ represents the alteration of the reloading yield stress after the path change $(g=1$ in its absence) and $h$ characterizes the work-hardening behaviour after reloading when compared with the one in simple strain path ( $h=1$ in this case). In this context the term $g / h \bar{\varepsilon}_{p}$ represents, in some way, the plastic prestrain value corrected for the path change. 
The above equation can have a formulation identical to that of the Swift law as it was originally formulated, for simple strain paths:

$$
Y\left(\varepsilon^{*}\right)=C^{*}\left(\varepsilon_{0}^{*}+\varepsilon^{*}\right)^{n},
$$

where $C^{*}=C h^{n}$ and $\varepsilon_{0}^{*}=\frac{g}{h}\left(\varepsilon_{0}+\bar{\varepsilon}_{p}\right)$. Experimental evidence allows to describe the strain hardening behaviour in reloading as shown in Fig. 1 (the reference curve, without prestrain, is also shown for comparison). In this figure $\sigma_{b e}$ (the stress at point $A$ ) is the back-extrapolated stress measured on the reloading curve $\left(\sigma_{b e}=\right.$ $\left.Y\left(\varepsilon^{*}=0\right)\right)$ and $\sigma_{r}$ is the reference stress measured at the same equivalent strain on the curve without prestrain $\left(\sigma_{r}=Y\left(\varepsilon=\bar{\varepsilon}_{p}\right)\right) . \quad\left(\sigma_{b e} / \sigma_{r}\right)$ is called the normalized reloading yield stress $[6,7,11,13-16] . \alpha$ represents a parameter that characterizes the influence of the strain path change on the work-hardening behaviour in reloading ( $\alpha$ is such that the two curves meet at point $B)$.

It is now possible to determine $h$ and $g$ as follows:

$h=\frac{\left(\varepsilon_{0}+\alpha n\right)-\left(\sigma_{b e} / \sigma_{r}\right)^{1 / n}\left(\varepsilon_{0}+\bar{\varepsilon}_{p}\right)}{\alpha n-\bar{\varepsilon}_{p}}$

and:

$g=\left(\sigma_{b e} / \sigma_{r}\right)^{1 / n}$.

The Eqs. (9) and (10) model the material behaviour in reloading for several complex strain paths. For that is necessary to know the monotonic behaviour, which allows us to determine the values of the parameters $C$, $n$ and $\varepsilon_{0}$, and to define the normalized reloading stress $\left(\sigma_{b e} / \sigma_{r}\right)$. The parameter $\alpha$ was found to be equal to 0.8 , in agreement with experimental results for copper and steel [19].

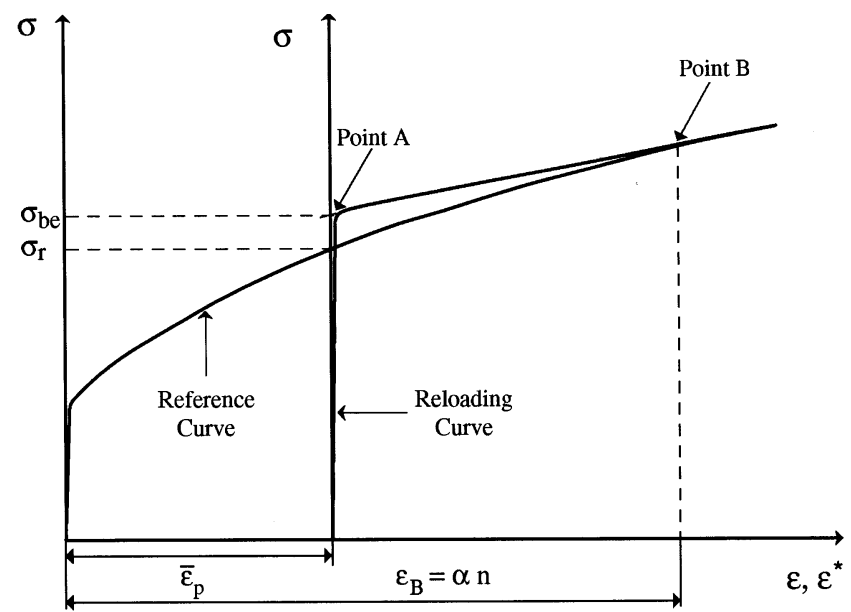

Fig. 1. Schematic representation of the true stress-true strain curves in strain without prestrain and after a prestrain $\bar{\varepsilon}_{p}$.
It can be shown that the above relations lead to an elastoplastic constitutive law, that relates the Jaumann derivative of the Cauchy stress tensor $\dot{\sigma}^{J}$ to the strain rate tensor $\boldsymbol{D}$ by an equation of the type:

$\dot{\boldsymbol{\sigma}}^{J}=\boldsymbol{C}^{e p}: \mathrm{D}$

where $\boldsymbol{C}^{e p}$ is a fourth order tensor designated by elastoplastic modulus [22].

The application of the constitutive model described above is limited to the reloading after sharp strain path changes. Indeed, because $\left(\sigma_{b e} / \sigma_{r}\right)$ must be previously known, the path change itself can not be part of the simulation. The simulated reloading strain path must be always the same than the experimental path used to calculate $\left(\sigma_{b e} / \sigma_{r}\right)$. Otherwise one needs more complex constitutive models that could predict $\left(\sigma_{b e} / \sigma_{r}\right)$ during the simulation, as those proposed by Teodosiu et al. [16].

\subsection{Time integration procedure}

Let $\Omega$ denote the region occupied by the sheet at a current time $t$. Assume that the boundary $\Sigma$ of $\Omega$ can be divided into two parts: $\Sigma_{1}$, where the rate of the nominal stress vector is prescribed and $\Sigma_{2}$, on which the velocity vector is prescribed. Then, the principle of virtual velocities traducing the incremental boundaryvalue problem goes as follows:

$\int_{\Omega} \sigma_{i j} \delta D_{i j} \mathrm{~d} \Omega=\int_{\Sigma_{1}} t_{i}^{*} \delta v_{i} \mathrm{~d} \Sigma$

with:

$\delta D_{i j}=\frac{1}{2}\left[\delta L_{i j}+\delta L_{j i}\right], \quad \delta L_{i j}=\frac{\partial\left(\delta v_{i}\right)}{\partial x_{j}}$

Eq. (14) is fulfilled for any virtual velocity field $\delta \boldsymbol{v}$ (with $\boldsymbol{\delta} \boldsymbol{v}=0$ on $\Sigma_{2}$ ) [23]. $\sigma_{i j}$ are the components of the Cauchy stress tensor with respect to the configuration at time $t, t_{i}^{*}$ are known functions of place and time.

The constitutive law given in Eq. (13) is formulated in stress velocities. However, in the principle of virtual velocities (Eq. (14)) is the total stresses that are in. So, it is necessary to linearize the principle (Eq. (14)) over the time interval $[t, t+\Delta t]$ which will depend on the type of time integration used and leads to the non-linear system:

$\mathscr{R}(\Delta \boldsymbol{u})=\mathbf{0}$,

where $\Delta \boldsymbol{u}$ is the displacement field for the current time interval [22].

To solve the non-linear system (Eq. (15)) for each time increment of the simulation we make use of an implicit algorithm of Newton-Raphson type. For details we refer to $[20,22,24]$. The global algorithm of the code is summarized in Table 1. 
Table 1

Global algorithm of the finite element code

\section{Repeat}

Calculate a tangent trial solution $d u^{0}$

Choose the trial increments by a $r_{\min }$ strategy [24]

Update the configuration

Repeat

Integrate the constitutive laws by using an implicit algorithm

Calculate the incremental displacements

Update the configuration

Until residual non-equilibrated forces are close to zero

Until the end of the process

\subsection{Finite element discretization}

In this paper we use the three-linear eight-node isoparametric hexahedron (for details see e.g. [25]). As full integration schemes lead to locking effects due to the plastic incompressibility requirement, we make use of a selective reduced integration technique. This procedure follows the formulation proposed by Hughes [26]. In brief, the dilatational contribution to the stiffness matrix is consider constant over the element and equal to its value at the central point of the element, whereas a full integration rule is used for the deviatoric part.

\section{Results and discussion}

A schematic representation of the tensile specimen to simulate the uniaxial sheet tensile test is shown in Fig. 2. This figure shows the actual specimen, and the symmetric quarter used in the finite element analysis. The thickness of the sheet is $1 \mathrm{~mm}$. The boundary conditions are also plotted in this figure and are specified at the boundary nodes as follows: the $x=0$ plane has no displacements in the $O x$ direction and the plane $y=0$ has no displacements in the $O y$ direction. The nodes in plane $x=L / 2$ have an imposed displacement in the $O x$ direction. Specimens with cross section variation are represented in Fig. 3. The dimension of the cross section variation is dictated by the parameter $a$. The finite element mesh consists of 235 hexahedrons with 576 nodes and is represented in Fig. 4 for the cases of $a=0 \mathrm{~mm}$.

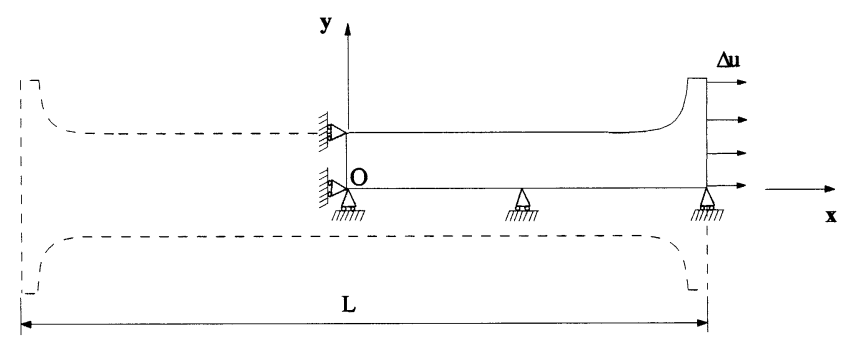

Fig. 2. Schematic representation of the specimen geometry and boundary conditions for the simulation of the tensile test.

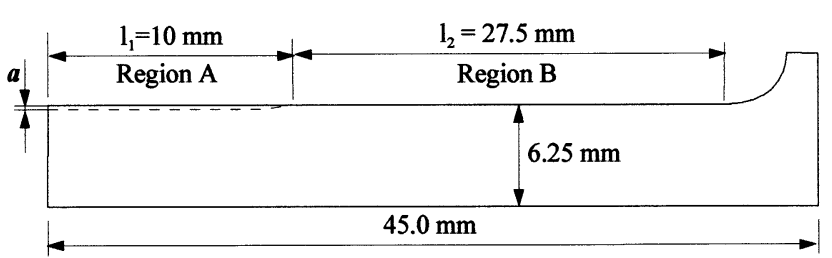

Fig. 3. Schematic representation of a quarter of the specimen with cross section variation. The value of $a$ was considered 0.05 and 0.1 $\mathrm{mm}$.

The first studied material used in this investigation was copper sheet with the following mechanical properties: Young modulus $E=119 \mathrm{GPa}$; Poisson ratio $v=$ 0.33 ; initial yield stress $Y_{0}=78.2 \mathrm{MPa}$; Swift coefficients: $K=461.6 \mathrm{MPa}$ and $n=0.35$. Those properties were obtained from tensile tests performed along the transverse direction (TD). The material had a slight plastic anisotropy with the following coefficients of anisotropy: $r_{0}=0.92, r_{45}=1.18$ and $r_{90}=0.54$, and were used to determine the constants of Eq. (5).

Sequential tension tests were performed by cutting ISO50 samples $\left(75 \times 12.5 \mathrm{~mm}^{2}\right)$ from large tensile specimens $\left(500 \times 220 \mathrm{~mm}^{2}\right)$ which had been previously strained up to three strain values $\left(\bar{\varepsilon}_{p}=0.06,0.12\right.$ and $0.18)$. The prestrain was obtained by pulling the samples along a direction doing $45^{\circ}$ with the rolling direction. After prestrain, IS050 samples were carefully cut, from the middle of the large strained specimen, along the transverse direction (used as reference direction). In all cases, the uniaxial tension tests were performed at room temperature. The initial strain rate was $5 \times 10^{-3}$ $\mathrm{s}^{-1}$ and the strain amounts were deduced from extensometer data.

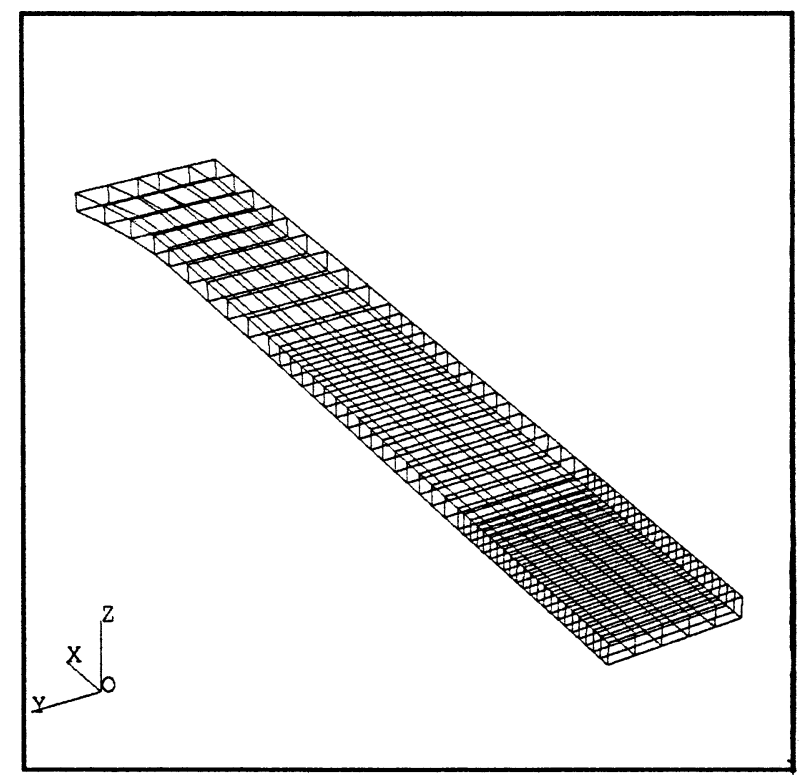

Fig. 4. Finite element mesh. 


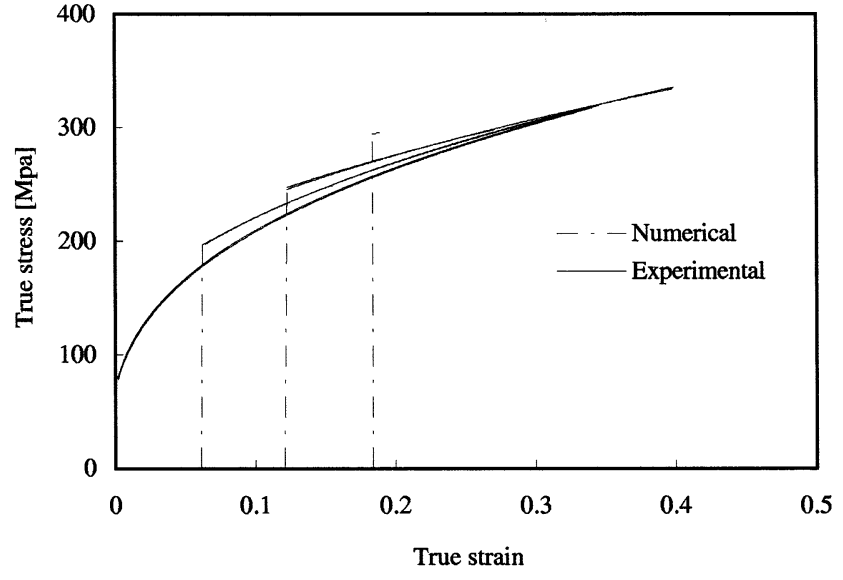

Fig. 5. Experimental and numerical stress-strain curves for cooper sheets during reloading in tension after prestrain values of 6,12 and $18 \%$. Stress-strain curves for samples without prestrain are also plotted.

The simulations correspond to the reloading in tension along the tranverse direction of the sheet, as for the experimental cases, represented by a normalized reloading yield stress $\left(\sigma_{\mathrm{be}} / \sigma_{\mathrm{r}}\right)=1.1$, determined from experimental data, which indicates a relatively 'strong' strain path change but not an extreme case $\left(\left(\sigma_{\mathrm{be}} / \sigma_{\mathrm{r}}\right)\right.$ can attain values dose to 1.15$)$ [7,11,13-15,17]. In Eq. (11), the $\alpha$ parameter is taken equal to 0.8 and three different prestrain levels were considered: $\bar{\varepsilon}_{p}=0.06,0.12$ and 0.18 . The choice of $\bar{\varepsilon}_{p}=0.18$ has the purpose to test the capacity of the code to simulate the early plastic instability for high prestrain levels.

Typical stress-strain curves that represent the material behaviour during reloading in tension, of cooper sheets, are shown in Fig. 5, corresponding to experimental and numerical results for specimens with constant cross section $(a=0)$. It can be observed that numerical results are very accurate. Plastic instability just after reloading is quite well predicted for the case of $\bar{\varepsilon}_{p}=0.18$.

Fig. 6 shows the true strain distribution along the longitudinal axes of the constant cross section sample, during deformation. Strain lines were plotted at displacement intervals of $1 \mathrm{~mm}$ except for the last line which is close to the maximum load. From $\bar{\varepsilon}_{p}=0$ to 0.18 , the number of lines decreases, showing a reduction in the failure time and total elongation. The development of strain localization can be compared from case-to-case, by observing the same strain line (counting from the bottom). In the sample without prestrain, the strain distribution is perfectly uniform, keeping the same characteristics during deformation. Strain localization occurs only after the maximum load is attained. To $\bar{\varepsilon}_{p}=0.06$ and 0.12 , the strain distribution is homogeneous at the beginning of the test, and the localization occurs at strain values close to the maximum load $\left(\varepsilon=0.26\right.$ to $\bar{\varepsilon}_{p}=0.06$ and $\varepsilon=0.15$ to $\left.\bar{\varepsilon}_{p}=0.12\right)$. The extension of the deformed region and its homogeneity is smaller to increasing values of prestrain. This is related to the change of work-hardening behaviour to each prestrain value. For $\bar{\varepsilon}_{p}=0.18$, the plastic instability occurs just at the beginning of reloading, which is a mechanical consequence of an important drop in the strain hardening rate at high levels of tension at the beginning of reloading.
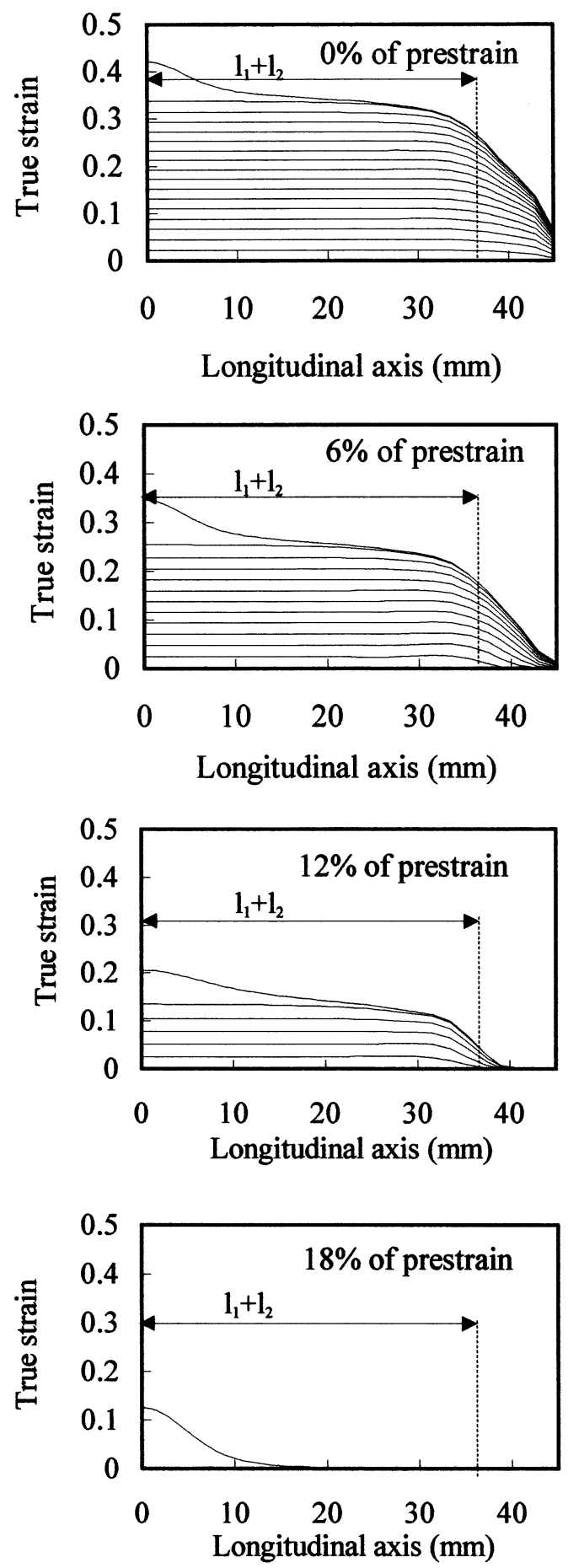

Fig. 6. Evolution of the strain distribution along the longitudinal axes for one region samples and for prestrain levels of $0,6,12$ and $18 \%$. 


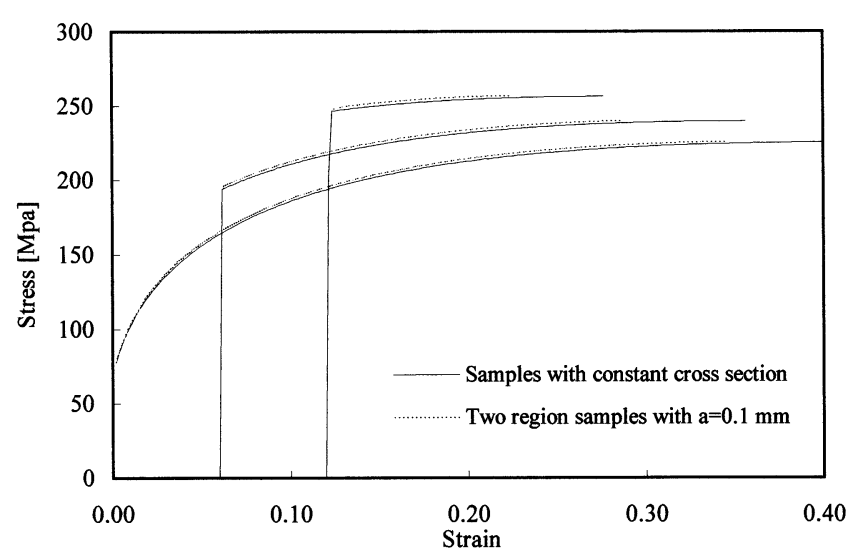

Fig. 7. Numerical stress-strain curves for samples without cross section variation and samples with $a=0.1 \mathrm{~mm}$, after prestrain values of 0,6 and $12 \%$.

The above results are related with specimens of constant cross section. This is not a real situation because experimentally prestrained samples presents always cross section variations. For example, variations in width, coming from machining, close to $0.1 \mathrm{~mm}$ were observed in tensile samples [17]. To emphasize the cross section variation effects several simulations were performed considering variations in width of $0.2 \mathrm{~mm}(a=$ $0.1 \mathrm{~mm}$ ) (Fig. 3), which are still lower than the maximum area fluctuations allowed by standardization, as for example, ISO50.

Fig. 7 shows the numerical stress-strain curves for all the situations tested. No significant differences are observed, but the residual homogeneous strain is smaller in the case of the samples with two regions, independently of the prestrain level. These values are given in Table 2.

Fig. 8 shows the true strain distribution in the two region samples. The prominent features of these curves can be summarized as follows:

- For the case without prestrain the deformation is homogeneous at the beginning of the reloading. However, contrarily to the case with constant cross section, it can be observed a non-uniform strain distribution even before the maximal load is attained.

- For the sample with 0.06 prestrain the behaviour is not very far from that in single path. The deformation is not very homogeneous, but it spreads over all

Table 2

Uniform residual deformation for samples with and without cross section variation for three levels of prestrain $(0,0.06$ and 0.12$)$

\begin{tabular}{|c|c|c|c|c|c|}
\hline \multicolumn{2}{|l|}{$\bar{\varepsilon}_{p}=0$} & \multicolumn{2}{|c|}{$\bar{\varepsilon}_{p}=0.06$} & \multicolumn{2}{|c|}{$\bar{\varepsilon}_{p}=0.12$} \\
\hline$a=0$ & $a=0.1$ & $a=0$ & $a=0.1$ & $a=0$ & $a=0.1$ \\
\hline $34.6 \%$ & $29.7 \%$ & $26.4 \%$ & $21.3 \%$ & $14.6 \%$ & $10.1 \%$ \\
\hline
\end{tabular}
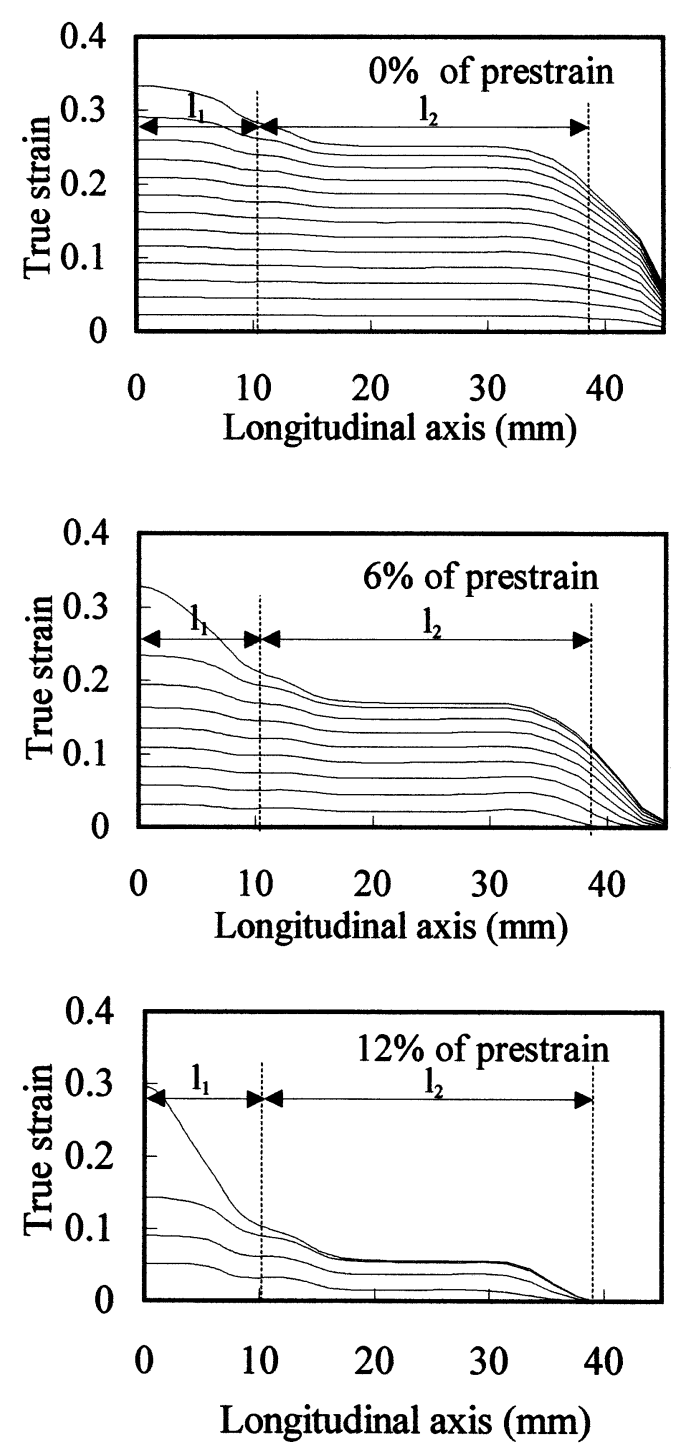

Fig. 8. Evolution of the strain distribution along the longitudinal axes for the two-region samples.

the specimen. The heterogeneity of deformation before the maximal load is attained is more pronounced than in single path, and it is possible to observe clearly the localization of the deformation in the middle of the sample in the last strain distribution line.

- In the test with $\bar{\varepsilon}_{p}=0.12$ the inhomogeneity of deformation along the axial direction starts earlier and develops more rapidly. The deformation clearly begins in the region A (Fig. 3), were the cross section is smaller. The final localization takes place with a strain level in the region B that is not greater than $5 \%$.

Analysis of the results in Fig. 6 (specimens with constant cross section) and Fig. 8 (two region specimens) shows evident differences in strain distribution characteristics. In fact, in the samples with constant cross section the strain distribution is perfectly homoge- 

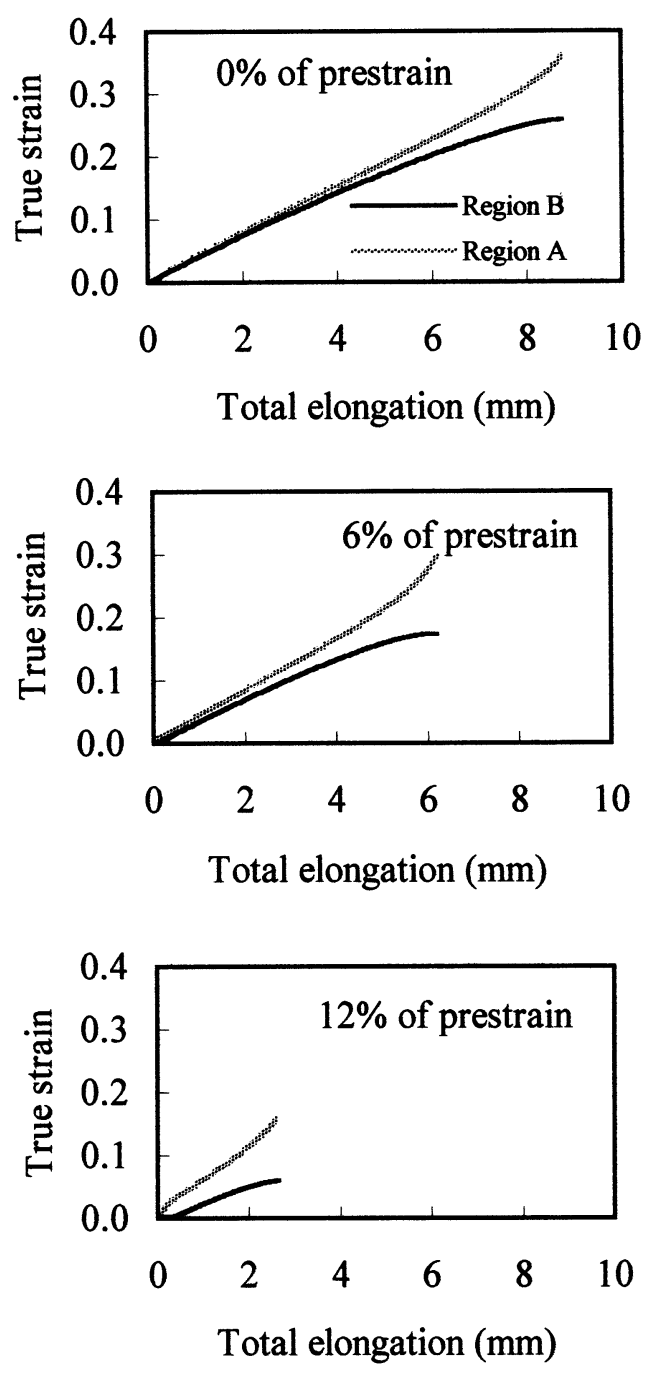

Fig. 9. Evolution of the true-strain in region A and B vs. the total elongation for the material with a hardening coefficient of 0.35 and $a=0.1 \mathrm{~mm}$.

neous over all the sample until the maximal load is attained; in this case the prestrain promote a slight reduction of the extension of the deformed region. The occurrence of inhomogeneities of the deformation at the beginning of the simulation is related to the existence of geometrical irregularities in the sample deformed area, as was observed in the simulations with the two region samples. This effect is more evident, as the prestrain value increases.

A detailed analysis of the evolution of the strain gradient along the axial directions of the two region specimens is possible with a graphic representation of the evolution of the true strain in each region, versus the total elongation. To calculate this strain distribution in the two regions, different lengths of measurement were considered $\left(l_{1}\right.$ and $\left.l_{2}\right)$ as shown in Fig. 3. Fig. 9 shows this evolution for the two region specimens without prestrain and with $\bar{\varepsilon}_{p}=0.06$ and 0.12 . To the sample in single path the deformation is clearly homo- geneous in the beginning of the deformation process. The differences in the average deformation in the two regions are no larger than 0.05 . For the 0.06 prestrained sample it is possible to observe differences in the strain gradient along the tensile axis since the beginning of the test. The differences in the average deformation in the two regions are, also in this case, no larger than 0.05 , except at the end of the test, close to the maximal load. In the last case ( 0.12 of prestrain), the differences are more pronounced. The deformation begins clearly in region $\mathrm{A}$. The deformation in region $\mathrm{B}$ begins only after $3 \%$ of deformation in region $\mathrm{A}$.

In order to understand the relation between the cross sectional area fluctuation and the hardening characteristics of the material, the same simulations were performed, but considering a copper sheet with lower hardening coefficient. The mechanical properties of this material are the following: initial yield stress $Y_{0}=184$ MPa, Swift coefficients: $K=440 \mathrm{MPa}$ and $n=0.26$.
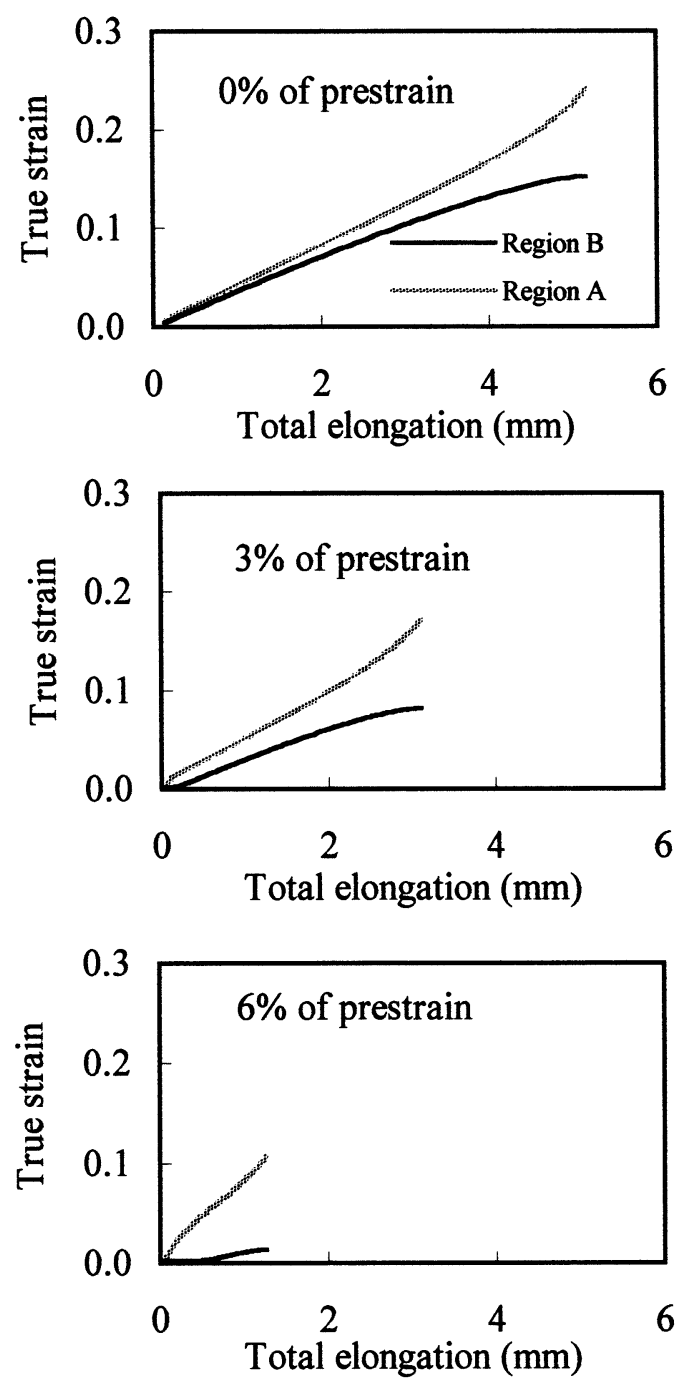

Fig. 10. Evolution of the true-strain in region $\mathrm{A}$ and $\mathrm{B}$ versus the total elongation for the material with a hardening coefficient of 0.26 and $a=0.1 \mathrm{~mm}$. 
The evolution of the average deformation in the two regions as a function of the total elongation is plotted in Fig. 10 for specimens without prestrain and for $\bar{\varepsilon}_{p}=0.03$ and 0.06 . It is clearly observed that for the case of $3 \%$ prestrain we have already a similar behaviour as for the case of $12 \%$ prestrain in the material with $n=0.35$. For $6 \%$ prestrain we have a very strong deformation gradient along the tensile axis. In fact we have an average deformation of $11 \%$ at the end of the process in the region with smaller section and only 3\% in region B. This behaviour indicates that the sensibility to the change of the cross section is more pronounced for materials with lower hardening coefficients.

A new set of simulations were also performed with the material with lower hardening coefficient, reducing the value a that defines the region A to $a=0.05 \mathrm{~mm}$. The correspondent results are plotted in Fig. 11. The strain distribution is substantially more homogeneous.
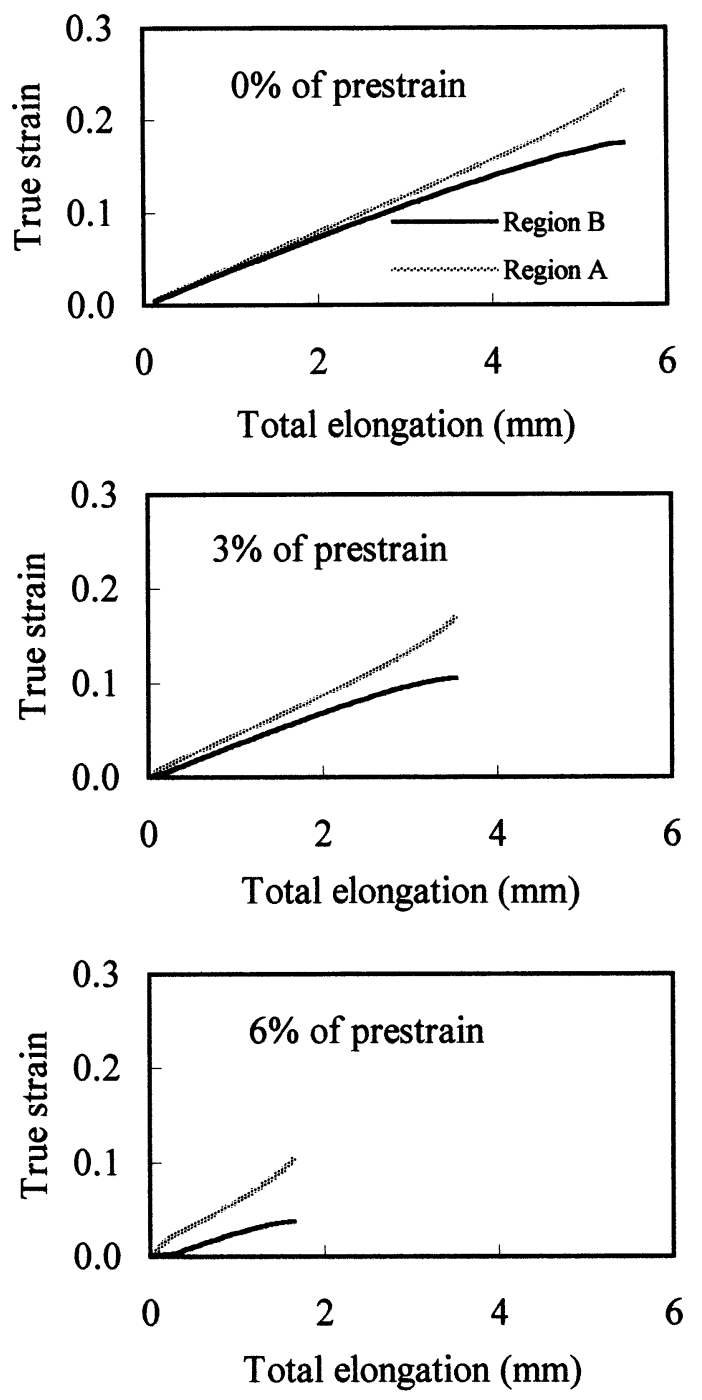

Fig. 11. Evolution of the true-strain in region A and B versus the total elongation for the material with a hardening coefficient of 0.26 and $a=0.05 \mathrm{~mm}$.
For the case of $6 \%$ prestrain the deformation still initiates only in region A but the effect of localization is less pronounced.

\section{Conclusions}

Finite element simulations of sheet tensile tests in samples with and without cross section variations have been performed using a modified Swift law that describes the material behaviour after a prestrain history.

The mechanical model corresponds to an orthotropic elastoplastic formulation with isotropic hardening modeled by the new equation. The evolution of the deformation process is described by using an updated Lagrangian scheme. The finite element code makes use of a fully implicit algorithm of Newton-Raphson type to solve the incremental problem. Spatial discretization is made with three-linear 8-node isoparametric 'brick' elements with a selective reduced integration technique.

The simulations presented here are in good agreement with experimental evidence which attest the good behaviour of the modified Swift law developed, and also the good performance of the finite element code used.

In constant cross section samples, the increasing of the prestrain level results in a reduction of the residual homogeneous strain and of the extension of the deformed region. The introduction of a cross section variation eliminates the homogeneity in the strain distribution and reduces the residual homogeneous strain (around 10\% for all the prestrain levels studied).

The results show that the strain distribution along the tensile axis in a prestrained sample depends on the level of the prestrain and also on the variations on the cross section which are inevitable in experimental prestrained samples. This dependence is more pronounced for materials with lower hardening coefficients where insignificant cross section variations (such as $50 \mu \mathrm{m}$ ) may produce severe heterogeneities in the strain distribution from the very beginning of the reloading.

\section{Acknowledgements}

The authors are indebted to JNICT for financial support trough FEDER and Programa PRAXIS XXI.

\section{References}

[1] A.K. Ghosh, W.A. Backofen, Metall. Trans. 4A (1973) 1113.

[2] D.J. Lloyd, H. Sang, Metall. Trans. 10A (1979) 1767.

[3] A.J. Ranta-Eskola, Met. Technol. 8 (1980) 45.

[4] R.H. Wagoner, Metall. Trans. 13A (1982) 1491.

[5] R.H. Wagoner, J.V. Laukonis, Metall. Trans. 14A (1983) 1487. 
[6] J.-H. Schmitt, E. Aernoudt, B. Baudelet, Mater. Sci. Eng. 75 (1985) 13.

[7] J.L. Raphanel, J.-H. Schmitt, B. Baudelet, Int. J. Plast. 2 (1986) 371.

[8] K. Chung, R.H. Wagoner, Metall. Trans. 14A (1987) 1487.

[9] A.B. Doucet, R.H. Wagoner, Metall. Trans. 20A (1989) 1483.

[10] D. Juul Jensen, N. Hansen, Acta Metall. Mater. 38 (1990) 1369.

[11] M.F. Vieira, J.-H. Schmitt, J.J. Grácio, J.V. Fernandes, J. Mater. Proc. Tech. 24 (1990) 313.

[12] D.V. Wilson, M. Zandrahimi, W.T. Roberts, Acta Metall. Mater. 38 (1990) 215.

[13] J.-H. Schmitt, J.V. Fernandes, J.J. Grácio, M.F. Vieira, Mater. Sci. Eng. A147 (1991) 143.

[14] J.V. Fernandes, J.J. Grácio, J.-H. Schmitt, in: J.L. Raphanel, F. Sidoroff (Eds.), Proceedings of the MECAMET' 91, A.A. Balkema, Rotterdam, 1993, p. 219.

[15] M.F. Vieira, J.V. Fernandes, J. Mater. Proc. Tech. 47 (1995) 261.

[16] C. Teodosiu, Z. Hu, in: S.-F. Shen, P. Dawson (Eds.), Proceedings of NUMIFORM' 95, A.A. Balkema, Rotterdam, 1995, p. 173.

[17] J.V. Fernandes, M.F. Vieira, Metall. Trans. 28A (1997) 1169.
[18] S. Hiwatashi, A. Van Bael, P. Van Houte, C. Teodosiu, Comp. Mat. Sci. 9 (1997) 274.

[19] J.V. Fernandes, D.M. Rodrigues, L.F. Menezes, M.F. Vieira, Int. J. Plast. 14 (1998) 537.

[20] L.F. Menezes, C. Teodosiu, A. Makinouchi, in: FE Simulation of 3D Sheet Metal Forming Processes in Automotive Industry, Tagungsbericht der VDI-Gesellschaft Fahrzeugtechnik, VDIVerlag, Dusseldorf 894 (1991) 381.

[21] C. Teodosiu, in: D.R.J. Owen, E. Hinton and E. Onate (Eds.), Proceedings of the Second International Conference on Computational Plasticity, Barcelona, 1989, Pineridge Press, Swansea, UK, p. 163.

[22] L.F. Menezes, PhD Thesis, Coimbra University, Portugal, 1995.

[23] R.M. McMeeking, J.R. Rice, Int. J. Solids Struct. 11 (1975) 601.

[24] C. Teodosiu, H.-L. Cao, in: D.R.J. Owen, E. Hinton, E. Onate (Eds.), Proceedings of the Second International Conference on Computational Plasticity, Barcelona, 1989, Pineridge Press, Swansea, UK, p. 959.

[25] G. Dhatt, G. Touzout, The finite element method displayed, Wiley, Chichester, New York, Brisbane, Toronto, Singapore, 1984.

[26] T.J.R. Hughes, Int. J. Num. Meth. Eng. 15 (1980) 1413. 